Historic, Archive Document

Do not assume content reflects current scientific knowledge, policies, or practices. 



\section{Summer Flowering ORCHIDS}

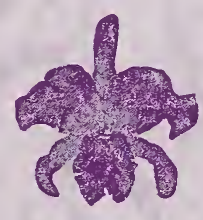

ORCHIDWOD: 830 PELHAMDALE AVENUE NEW ROCHELLE, NEW YORK 


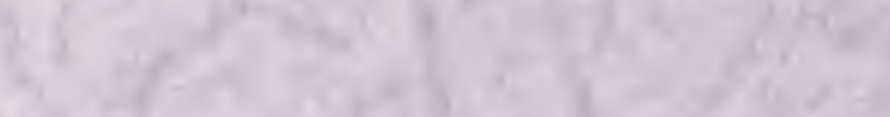

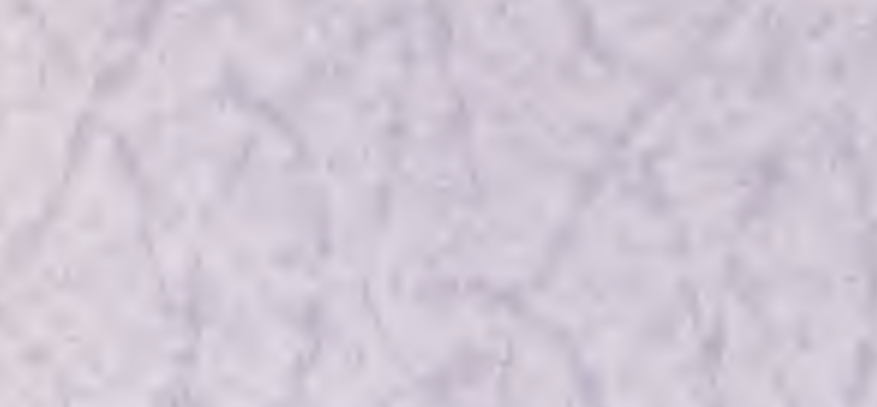

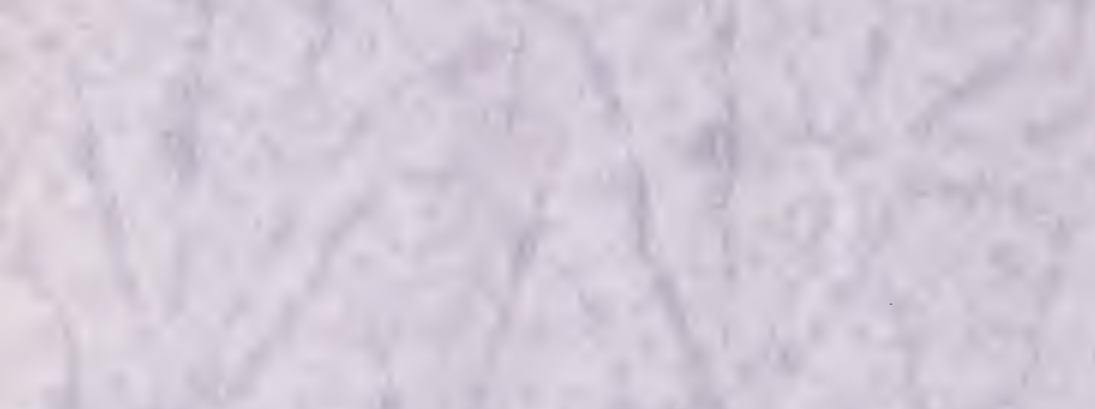

-

$\sqrt{2}+x^{2}$

(1)
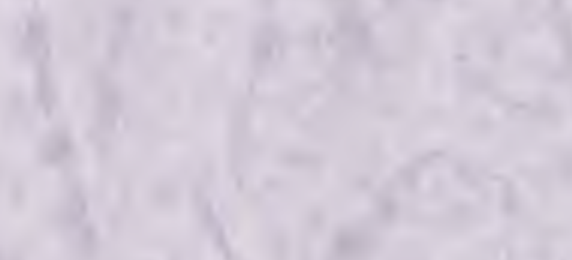

$+\frac{1}{4}+x$

$x^{2}$

$x^{2}-x^{-1}$

12.

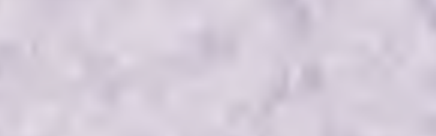

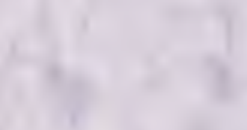

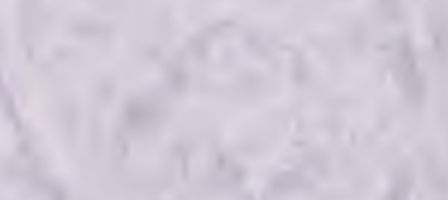

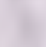

$+$

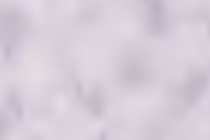

$\sqrt{2}$

$1+2=$

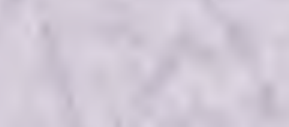

ra
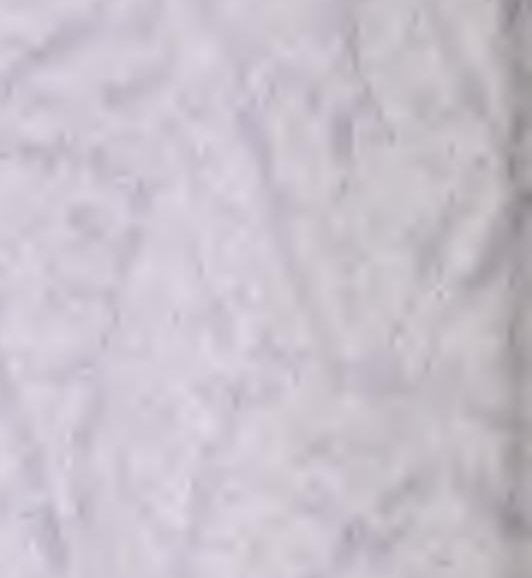


\section{INTRODUCTION}

any of our finest varieties of Orchids come into bloom during the summer months when many other flowers are available and Orchids for cut flowers are not in such great demand. Therefore we are offering the following plants which are good subjects for a private collection of Orchids at very reasonable prices. The special and fine varieties have great value in that they have been proven as makers of other good hybrids.

With the engagement of the exclusive services of Mrs. D. A. Linder, one of the world's leading experts on Orchid seed germination by the Fungus Method, we can now offer this service to the private as well as the commercial grower. The danger of heavy losses of seedlings incidental to other methods of Orchid seed germination will be greatly lessened since the Fungus Method has been proven to produce fastergrowing, sturdier seedlings than those obtained by any other method.

Recent additions at Orchidwood include a large and a small showhouse in the style of private conservatories, and a large, comfortably furnished reception room. A representative group of plants in bloom is always on display, and visitors are assured of a cordial welcome at all times.

ORCHIDWOOD, INC. New Rochelle, N. Y. 



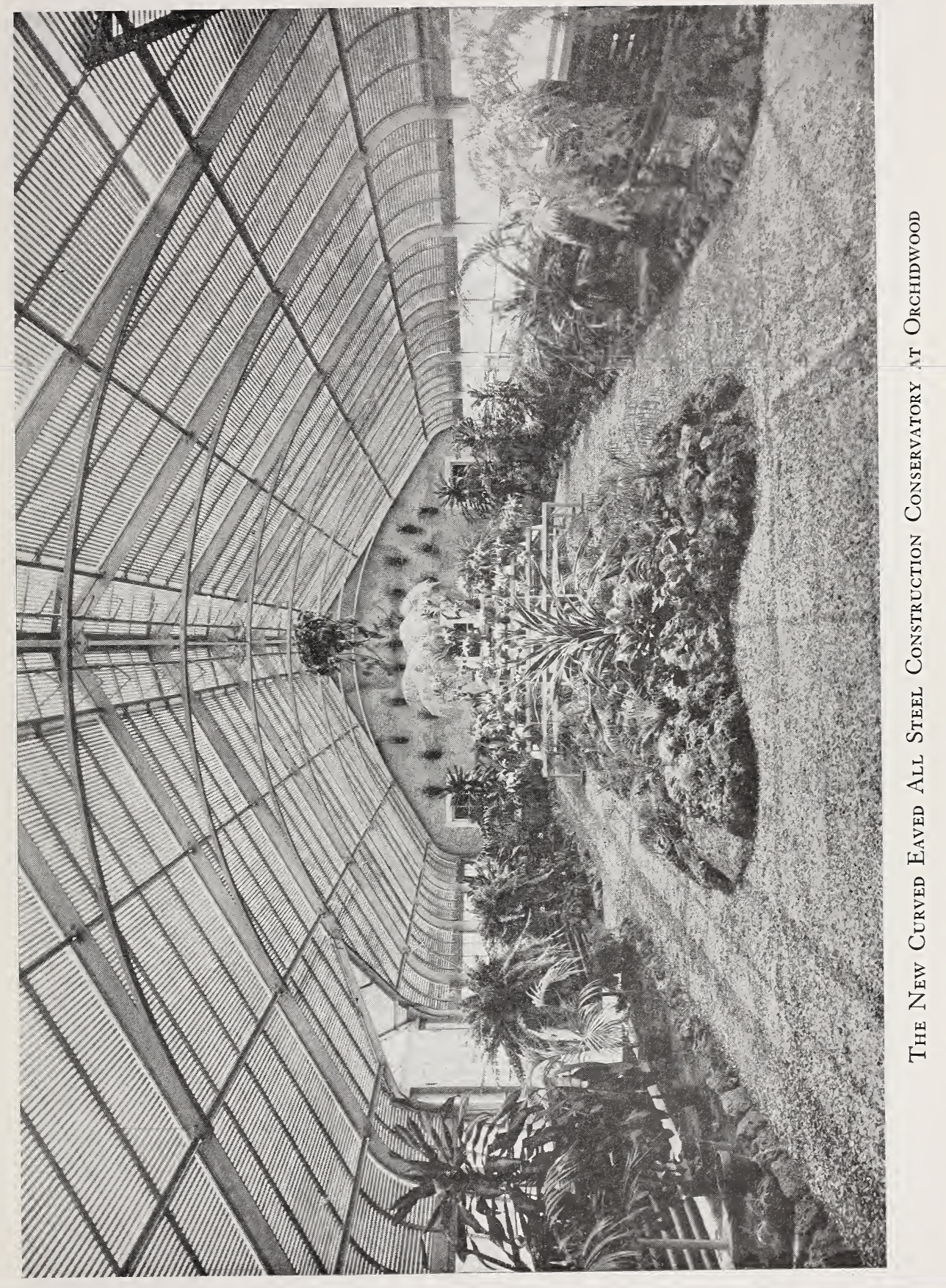




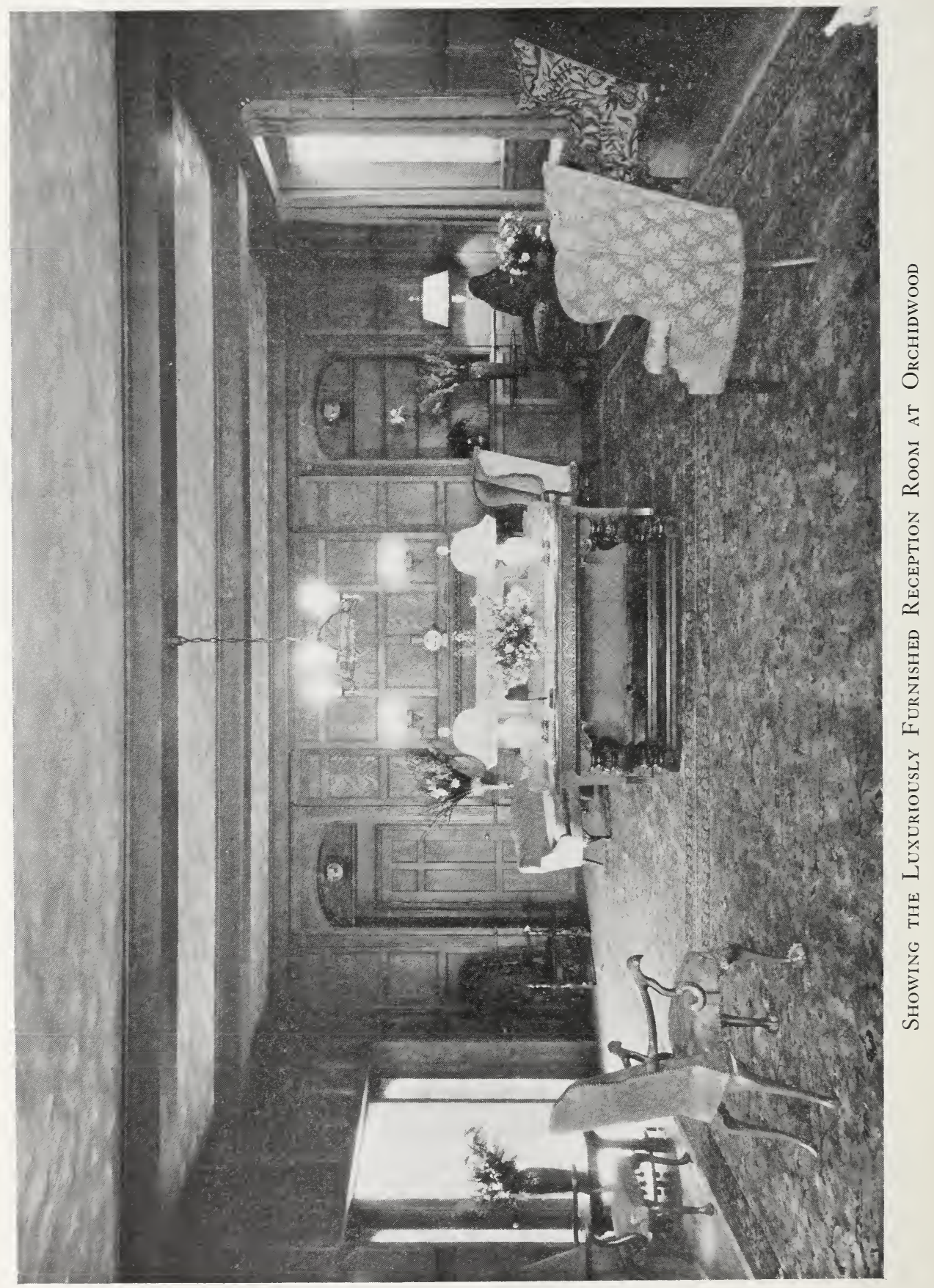


All the following plants are in a strong, healthy condition and have flowered or will flower during the summer months. Plants purchased from this list that bloom at any other time will be replaced by plants that will flower during the summer. Average size five to seven bulbs, one to two leads.

Brassavola Digbyana-One of the parents of most of the primary Brassocattleyas

BRASSOCATTLEYA

Heatonensis (B. Digbyana $\times$ C. Hardyana). Lavender color, deeply fringed lip

John Linford (C. Prince Shimadzu $\times$ B. C. Rosita). One of the finest modern hybrids

Maronae (B. Digbyana $\times G$. Gigas)

Nestor (B. C. Maronae $\times$ C. Iabiata). Large, well-balanced flowers

Special dark variety

Olbia (B. C. Leemann $\times$ C. Mrs. Pitt). Flowers nearly pure white

Veitchi (B. Digbyana $\times$ C. Mossiae $)$

20.00

50.00

20.00

25.00

35.00

35.00

20.00

\section{Brassolaelia}

Helen (B. Digbyana $\times$ L. tenebrosa). Thin petals, wide fringed lip

Veitchi (B. Digbyana $\times$ L. purpurata).

I0.00

$5 \mathrm{I} .00$

BrassolaELIOCATTLEyA

Callisto (B. Veitchi $\times L$. C. callistoglossa)

20.00

Everest (B. C. Leemann $\times$ L. C. Canhamiana). Extra large flowers, fine fringed lip

Heliolata (B. L. C. Maculata $\times$ C. Heliodor). Fine Variety -Orange yellow sepals and petals; deep purple lip; flower of fine shape

Lady Diana (B. L. Veitchi $\times$ C. Dowiana)

The Baroness (B. C. Leemann $\times$ L. C. Ophir). Parent of many fine yellow hybrids

30.00

250.00

15.00

75.00

Cattleya Species

Bowringiana

\$5.00. Larger plants 7.50 to 10.00

Dowiana-Very popular for hybridization

20.00

Gaskelliana-Free flowering

10.00

gigas-One of the best species of Cattleya 
Alcimeda alba (Gaskelliana alba $\times$ Labiata Purity). Pure white flowers of good size and shape

Amabilis (labiata $\times$ gigas). Large dark flowers on strong growing plants

Armstrongiae (Hardyana $\times$ Loddegesi)

25.00

Ashtoni (Harrisoniana $\times$ gigas)

I0.00

Boadicea (Gaskelliana X Hardyana)

I0.00

Comet (Dowiana $\times$ Warneri Var. Edward H. Roehrs). One of the darkest Cattleya hybrids; exclusixe Orchidwood stock

Cybele (Gaskelliana X Lueddemanniana)

Alba-A fine all white flower with broad petals

Dionysius (Fabia $\times$ gigas). Dark variety

Alba-Broad, white sepals and petals; deep purple lip

Dupreana (gigas $\times$ Warneri). Extra large flowers, good color and shape

20.00

Emily (Enid alba $\times$ Hesta). White sepals and petals, purple lip

20.00

Enid (gigas $\times$ Mossiae). One of the largest flowering hybrids $\quad$ I5.00

Fabia (Dowiana $\times$ labiata)

I 5.00

Special Variety

25.00

Fine Variety-Extra dark, large flowers

50.00

Freya (Dowiana $\times$ Mantini)

I 2.50

Gay Gordon (Hardyana X Van Houtte)

20.00

Good Variety-Dark flowers of good shape

35.00

Gildeni (Hardyana X Maggie Raphael)

I5.00

Gloriette (Tityus $\times$ Hardyano-Warneri). A wonderfully shaped flower of good color and texture; ideal for hybridization

Fine Variety-similar to L. C. Titymoma F. C. C.

Hardyana (Dowiana $\times$ gigas)

Good Variety-Attractive contrasting color

Fine Variety-All dark flower

Alba-White sepals and petals; purple lip

Alba-Special Variety-Unusually dark lip

Alba-Fine Variety-One of the finest types of this cross obtainable 
Harold (Gaskelliana X gigas)

IO.0O

Special Variety-Large flowering type

20.00

Helen Langley (Undine $\times$ Myra Peeters alba). One of the purest white Cattleyas

35.00

Heliodor (irridescens $\times$ Venus). Yellow sepals and petals; purple, cut lip

Var. Orchidwood-Magnificent orange-yellow sepals and petals; royal-purple full lip; flower of fine shape and exceptional texture

Hentschelli (Dupreana $\times$ gigas)

Good Variety-Very large flowers of good color

25.00

H. S. Leon (Schroederae $\times$ gigas). A vigorous grower, free blooming, with well-shaped flowers of soft pink color

Iris (bicolor $\times$ Dowiana). Cut-lip type

20.00

Lord Rothschild (Dowiana X Gaskelliana). Extra dark Variety

IO.0O

Malcolm Canmore (Armainvillierensis $\times$ Lueddemanniana)

25.00

Mantini (Bowringiana $\times$ Dowiana)

I 5.00

Miss Kemp (Bowringiana $\times$ Fabia). One of the best types of the small hybrids; flowers of fine shape and deep purple color; five to ten flowers on stem

IO.00

Miss M. Armstrong (Dowiana X Helen Langley). Varigated, unusual colors in lighter shades; fine shape and heavy texture

I5.00

Mrs. Myra Peeters (Gaskelliana alba $\times$ Warneri alba). One of the most consistent June flowering white hybrids; large flowers of good shape

Portia (Bowringiana $\times$ Labiata) \$7.50. Larger plants

Pretoria (Dowiana $\times$ Peetersi). An excellent, well-balanced flower of good color, shape and texture; free flowering

I5.00

Extra dark Variety

25.00

Prince John (Dowiana $\times$ Hardyana alba). Creamy white sepals and petals, purple lip with beautiful golden yellow veins

Special Variety-Yellow sepals and petals similar to Catt. Hardyana Var. Clement Moore 
Princess Royal (Fabia $\times$ Hardyana $)$. Dark Variety

Alba-(Fabia alba $\times$ Hardyana alba $)$

Aureata-(Fabia alba $\times$ Hardyana alba Var. Clement

Moore). Golden yellow sepals and petals; broad royal purple lip of heavy, velvety texture

100.00

Priscilla (Enid $\times$ Lueddemanniana)

20.00

Quibo (Dowiana $\times$ Miss Williams). Attractive, light colored flowers of extra heavy texture and good shape

I5.00

Santa Monica (Mendeli $\times$ Lord Rothschild). Flowers greatly resemble Catt. Tityus

Sir Walter Scott (Harold $\times$ gigas $)$

Snowdon (Labiata alba $\times$ Suzanne Hye). All white flowers of good shape and texture; a vigorous grower

Sylvia (Dowiana $\times$ Fabia). Extra dark Variety-Rich purple thruout

Alba-Creamy white sepals and petals

Tityus (Enid $\times$ Octave Doin)

Special Variety-Parent of many fine hybrids

Triumphans (Dowiana $\times$ Rex)-Roehrs Variety

Van Houtte (Lord Rothschild $X$ gigas). Large flowers of good color and shape.

Extra dark Variety

20.00

I 5.00

50.00

25.00

25.00

25.00

50.00

35.00

20.00

35.00

Venus (Dowiana $\times$ Iris). Cut lip type

I0.00

Full lip type; bronze sepals and petals

20.00

Full lip type; yellow sepals and petals

Veiris (Venus $X$ Iris). Cut lip, yellow petals

35.00

Special Variety-Fine yellow petals

15.00

50.00

Fine Variety-Full lip, yellow petals

I00.00

Vestalis (Dowiana $\times$ Maxima)

$7 \cdot 50$

Cypripedium

Lawrenceanum (species)

Maudiae (callosum Sanderae $\times$ Lawrenceanum). One of the most free-flowering and easily propagated varieties; flowers green and white

Dendrobium Phalaenopsis (Species)

I0.00

EPIDENDRum ciliare (Species) 
tenebrosa (Species)-has true bronze sepals and petals which carry over to its hybrids

I5.00

\section{LAELIOCATTLE.A}

A. C. Burrage (L. C. Herscentia $\times$ C. Dowiana) Special Variety-Bronze sepals and petals

20.00

35.00

Aphrodite $(C$. Mendeli $\times$ L. purpurata) Flowering size Larger plants, very strong growers

I 2.50

$\mathrm{I} 7.50$

Arran (L. C. Australia $\times$ C. Gaskelliana). Sepals and petals have soft bronze tint

Arturo (L. C. Hyeana $\times$ C. gigas)

Good Variety-A deep purple flower

Athene (callistoglossa $\times$ St. Gothard). Very large flowers of fine color and texture

Attraction (luminosa $\times$ Myrrah). One of the best of the bronze tinted varieties with deep-purple lip

Fine Variety-Good bronze sepals and petals; intensely deep rich purple lip with bright golden-yellow side lobes

Bletchleyensis (C. gigas $\times$ L. tenebrosa). Creamy white petals, purple lip

Dark bronze sepals and petals

I 2.50

20.00

Britannia (C. gigas $\times$ L. C. Canhamiana). Large flowers of good shape and color

35.00

35.00

I00.00

Caledonia (C. Mendeli $\times$ L. C. Bletchleyensis). Another of good bronze petal types

callistoglossa (C. gigas $\times$ L. purpurata $)$

Extra strong plants

Fine large plants, special dark variety

Cameronian (L. C. Dominiana $\times$ C. Princess Royal)

20.00

Extra dark variety

Alba-White sepals and petals, purple lip

I 5.00

25.00

35.00

I 5.00

25.00

25.00

Canhamiana ( C. Mossiae $\times L$. purpurata). One of the best June hybrids; well balanced flowers on long, thick stem Extra dark variety

I5.00

25.00

Colmaniana (C. Dowiana $\times$ L. C. callistoglossa)

20.00

Cortina ( C. Empress Frederick $\times$ L. C. callistoglossa)

I 5.00

Special Variety-Extra dark flowers of good shape 
eximia ( $C$. Warneri $\times$ L. purpurata). Vigorous grower; large flowers, deep lip

G. G. Whitelegge (C. Hardyana X L. C. callistoglossa) .

15.00

Grisette (eximia $\times$ Golden Oriole) Flowering size

10.00

Special Variety-Yellow tint in sepals and petals

Jacquinetta (E. Emp. Frederick $\times$ L. C. Lustre). One of the finest Laeliocattleyas; large flowers of good shape and striking colors

Jas Goodier (L. C. Canhamiana X C. Emp. Frederick)

Large, bold flowers of rich colors

25.00

Martineti (C. Mossiae $\times$ L. tenebrosa)

20.00

Special Variety-Deep bronze sepals and petals; royal purple lip

I5.00

Massasoit (L. C. Nella $\times$ C. Hardyana rubra)

Good Variety-Large, dark flowers

Fine Variety-Bronze sepals and petals; dark purple lip

Var. Orchidwood-One of the darkest of all laeliocattleyas; extra fine lip

Mrs. Harold Lloyd (L. C. Aphrodite $\times$ C. Van Houtte).

Fine round flowers of good color

Special Variety-Excellent shape and color

Mrs. M. A. House (C. Hardyana X L. C. Golden Oriole)

25.00

15.00

35.00

50.00

75.00

Good Variety-Yellow sepals and petals

20.00

35.00

15.00

35.00

Mrs. Medo (L. C. Luminosa $\times V$. Venus)

25.00

Special Variety-Attractive bronze petals

35.00

Fine Variety-Flower of extremely heavy texture; bronze

sepals and petals, crimson lip

50.00

Phoenix (C. Dowiana $\times$ L. C. Henry Greenwood)

20.00

Portita (L. C. Callistoglossa $\times$ C. Portia)

I0.00

Profusion (L. C. Serbia $\times$ C. Hardyana)

25.00

Special Variety-Fine all-round flowers

Rabeiana (C. Dupreana $\times$ L. purpurata). Large flowers of good color

50.00

Thurgoodiana (C. Hardyana $\times$ L. C. Martineti)

20.00

Special Variety-Fine color thruout

25.00

Wellesleyi (C. gigas $\times$ L. C. Martineti) .

50.00

25.00

Special Variety-E. H. Roehrs type

50.00 
Miltonia Bleuana (vexillaria $\times$ Roezli). Large flowers

St. Andre (Bleuana $\times$ Roezli)

Oncidium altissimum (Species). Very long sprays of yellow flowers blotched with brown

flexuosum (Species). Graceful, branched sprays of pure yellow flowers

IO.OO

Sobralia macrantha (Species)

Sophrolaeliocattleya

Baldwini (C. gigas $\times$ S. L. C. Meuse)

Fine Variety-Very dark flowers of good shape

Mrs. F. Mercer (C. Hardyana X S. L. C. Meuse)

Mrs. G. E. Baldwin (L. purpurata $\times$ S. L. C. Meuse)

I 5.00

Extra dark variety; fine shape

Thelma (C. Lady Richmond X S. L. C. Isabella)

20.00

35.00

25.00

VAnda Teres (Species). Good sized, rose colored flowers on short spray

Zygopetalum Discolor (Species). One of the more interesting and desirable botanical types

Osmunda Fibre-A very fine grade of clean, firm compost, picked ready to use-Large Bag

\section{ORCHID COLLECTIONS}

25 Plants in Variety

50 Plants in Variety

Ioo Plants in Variety
$\$ 200, \$ 300, \$ 500$

\$350, \$ 500, \$1000

\$600, \$1000, \$1750

Special Quotations On Large Groups

ORCHIDWOOD, INC.

New Rochelle, N. Y. 




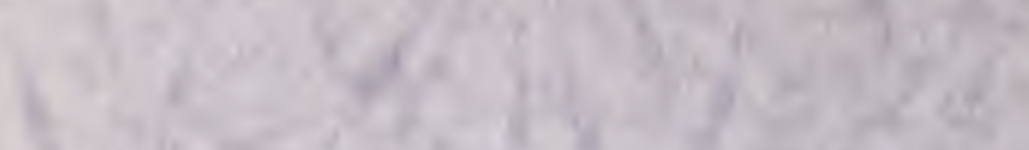

西

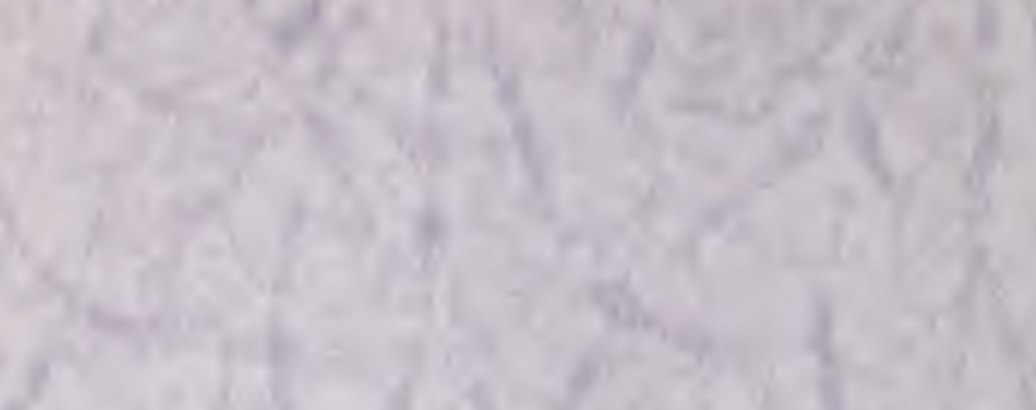

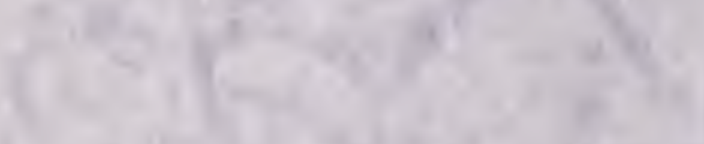

I.

$\sqrt{1}$

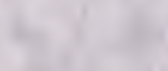

1)

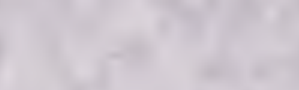

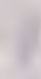

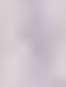

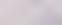

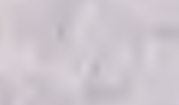

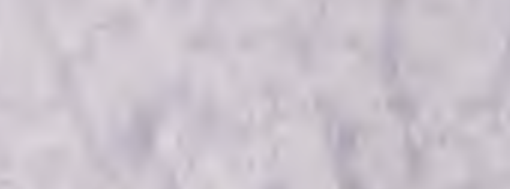

3.

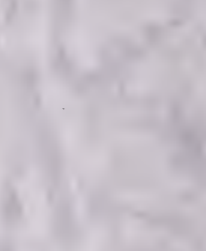

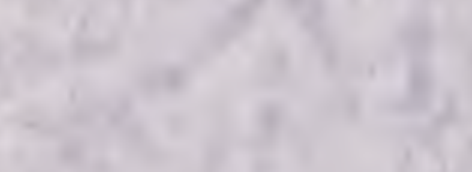

8

(2)

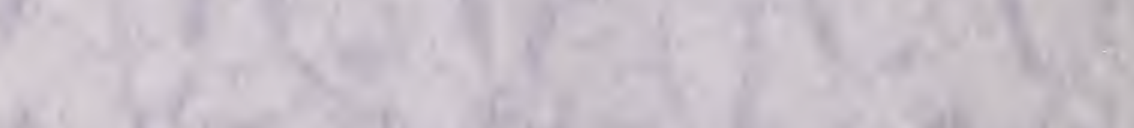

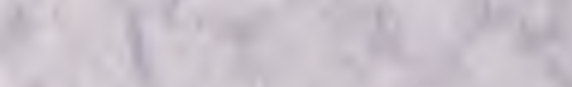

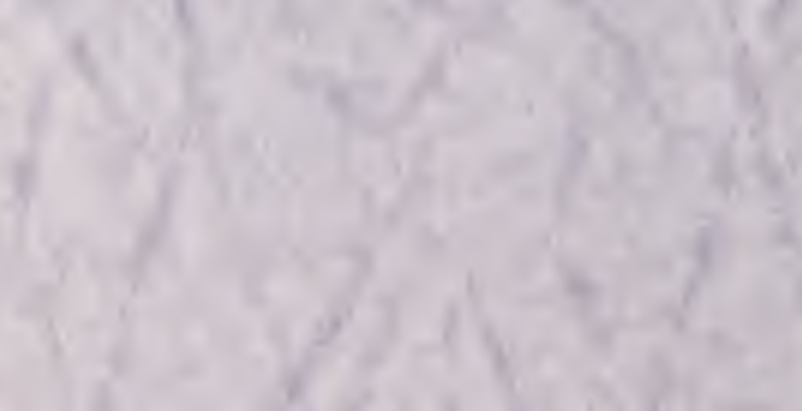

$\sqrt{1+20}$

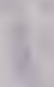

(1)

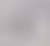

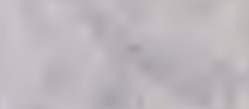

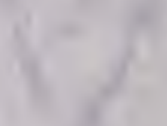

